\title{
Visual-auditory Volume Rendering of Dynamic Quantum Chemistry Molecular Fields
}

\author{
Evgeniya Malikova $^{1}$, Valery Adzhiev ${ }^{1}$, Oleg Fryazinov $^{1}$ and Alexander Pasko ${ }^{1,2}$ \\ ${ }^{1}$ Bournemouth University, Bournemouth, U.K. \\ ${ }^{2}$ Skolkovo Institute of Science and Technology, Moscow, Russia
} $\begin{array}{ll}\text { Keywords: } & \text { Volume Rendering, Visual-auditory Ray-casting, Signed Distance Fields, Multi-scale Visualisation, Molecular } \\ & \text { Visualisation. }\end{array}$

Abstract: This work deals with a visual-auditory visualisation of dynamic heterogeneous objects represented by continuous scalar fields obtained from quantum chemistry. The research concentrates on complex phenomena modelling and rendering aspects and takes advantage of GPU implementation. The approach uses the constructive HyperVolume for the multi-scale representation of the molecular phenomena. To propose an approach to the visual-auditory rendering, we adapt the real-time interactive volume ray-casting to compute the optical and auditory properties. We demonstrate the approach application for the visual-auditory rendering of dynamic molecular structures.

\section{INTRODUCTION}

As computer simulations or experimental studies are involved, scalar fields are used in many research areas such as computational chemistry, medical data analysis and physical phenomena studies. We consider an example of the analysis of the molecular structures that are dynamic objects with internal properties and geometry changing in time. As the detailed and accurate study of the properties of molecular structure can be computationally expensive, the analysis of the molecular structures benefits from simultaneous use of representations of various objects' properties (Wahle and Wriggers, 2015) that are obtained with different computational procedures. We consider a particular case study of dynamic molecular structures obtained by the quantum chemistry simulation.

The modelling, rendering and visual analysis of such multi-scale representations of the phenomena are not straightforward. In Volume Rendering the problem is addressed with the use of Multidimensional Transfer Functions (TF) (Kniss et al., 2003) and optical models with complex object-light interactions (Wahle and Wriggers, 2015). Those techniques are used to improve the quality of visual analysis and to highlight features of interest. However, limitations of the visual perception can not be avoided solely with the enhancement of the optical model. The visual system can be overloaded and perturbed due to the fatigue of the user. An introduction of auditory sensory stimuli is a well-known technique that is used to address a problem of visual analysis limitations (Hermann et al., 2011). For scalar data analysis problem, the works (Lodha et al., 1997) and (Gionfrida and Roginska, 2017) introduce the auditory analysis to address the problems, where the visual analysis fails.

We suggest a simultaneous visual-auditory analysis as a tool to address a problem of molecular structures complexity for a particular problem of the results of quantum chemistry problem study. The main contributions of this work are the following:

1. We propose a unified approach to the visualauditory study of the physical phenomena the molecular fields based on ray-casting.

2. We take advantage of HyperVolume (HV) (Pasko et al., 2001) model for scene representation as a dynamic heterogeneous object with visual and auditory properties.

3. We consider the case study of the molecular structure, that is a result of quantum simulation, to perform simultaneous visual-auditory analysis to get insight into the multi-scale representation of the phenomena.

We demonstrate how the GPU implementation of the constructive approach for HV scene can be used to efficiently perform simultaneous visual-auditory analysis on the base of the ray-casting procedure. 


\section{BACKGROUND AND RELATED WORKS}

\subsection{Volume Rendering of Scalar Fields}

A scalar field is a mapping $f: x \rightarrow \mathfrak{R}, x \in \mathfrak{R}^{n}$, which associates any point in space with a scalar value. Visualisation of scalar fields usually employs Volume Rendering technique that operates the initial data as volumes that are stored in the texture memory.

The conventional Volume Rendering scheme suggests the volume boundary (or scalar field domain) is defined by a bounding box. The ray/volume intersection is computed to define the ray's start and end points and after the equations 1,2 are solved with the ray-marching procedure (Fig. 1 (a)).

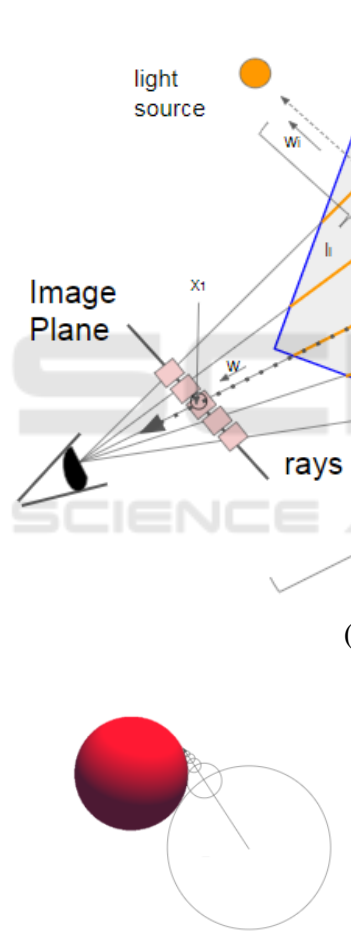

(b)

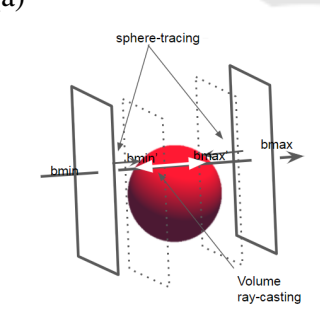

(c)
Figure 1: Volume Rendering a) Schematic representation of basic ray tracing for Volume rendering; b) Sphere-tracing scheme (Hart, 1996); c) SDF accelerated Volume Rendering on basis of sphere-tracing scheme.

In Volume Rendering a simple model of light interaction with an object considers only emission and absorption (Jönsson et al., 2014):

$$
\begin{aligned}
& L\left(\overrightarrow{x_{1}}, \vec{\omega}\right)=T\left(\overrightarrow{x_{0}}, \overrightarrow{x_{1}}\right) L\left(\overrightarrow{x_{0}}, \vec{\omega}\right)+ \\
& \qquad \int_{\overrightarrow{x_{0}}}^{\overrightarrow{x_{1}}} T\left(\vec{x}_{s}, \overrightarrow{x_{1}}\right) E\left(\vec{x}_{s}\right) d \vec{x}_{s}
\end{aligned}
$$

where $L\left(\overrightarrow{x_{1}}, \vec{\omega}\right)$ is an intensity at the point $x_{1}$; $T\left(\vec{x}_{s}, \overrightarrow{x_{1}}\right)=e^{-\int_{\overrightarrow{x_{s}}}^{\overrightarrow{x_{1}}} \sigma_{t}(\vec{x}) d \vec{x}}$ is an attenuation of light as it travels from the point $x_{s}$ to $x_{1}$ defined in the general case by an extinction coefficient $\sigma_{t}\left(\vec{x}_{1}\right)=$ $\sigma_{a}\left(\overrightarrow{x_{1}}\right)+\sigma_{s}\left(\overrightarrow{x_{1}}\right)$ that takes into account absorption and scattering (not considered in this model); $E\left(\vec{x}_{s}\right)$ is a light contribution at the point $x_{s}$, in other words, the term describing emission process that can be written as $\sigma_{a}\left(\vec{x}_{s}\right) * L_{e}\left(\vec{x}_{s}, \vec{\omega}\right)$

The extended equation takes into account a single light scattering (Max and Chen, 2010) and is also considered for an interactive Volume Rendering (Jönsson et al., 2014):

$$
\begin{aligned}
L\left(\overrightarrow{x_{1}}, \vec{\omega}\right)= & T\left(\overrightarrow{x_{0}}, \overrightarrow{x_{1}}\right) L\left(\overrightarrow{x_{0}}, \vec{\omega}\right)+ \\
& \int_{\overrightarrow{x_{0}}}^{\overrightarrow{x_{1}}}\left(\sigma _ { a } ( \vec { x _ { s } } ) * L _ { e } \left(\overrightarrow{x_{s}}+\sigma_{s}\left(\vec{x}_{s}\right) *\right.\right. \\
& \left.\left.L_{s s}\left(\overrightarrow{x_{s}}, \vec{\omega}\right)\right) T\left(\vec{x}_{s}, \vec{x}_{1}\right)\right) d \vec{x}_{s}
\end{aligned}
$$

where $L_{s s}\left(\vec{x}_{s}, \vec{\omega}\right)$ represents the scattered radiance in the direction $\vec{\omega}$.

The shading and depth perception can provide an addition to $3 \mathrm{D}$ shape cues and thus enhance visual analysis and highlight the features of interest (Kniss et al., 2003). The Blinn-Phong model (Levoy, 1988) was an initial enhancement of the classical emissionabsorption model 1. This model is widely used in Volume Rendering to highlight features of interest such as edges (Jönsson et al., 2014), although it was designed for shading the surfaces and can produce dark regions (Kniss et al., 2003).

Complex optical model computation like in the equation 2 allows to significantly enhance visual analysis quality (Wahle and Wriggers, 2015), however, are still computationally expensive (Jönsson et al., 2014) for the interactive and dynamic Volume Rendering.

Computation of complex optical models for interactive Volume Rendering takes advantage of modern graphics hardware (Schlegel et al., 2011). The modern algorithms often employ the idea of splitting the optical model computation into separate parts, mainly concerned with the lighting conditions that can be precomputed into textures independently and updated on-demand (Kniss et al., 2003), (Schlegel et al., 2011).

The other tool to address the complexity of studied phenomena in Volume Rendering is the Multidimensional Transfer Function (TF) (Kniss et al., 2003). TFs are used to address a problem of simultaneous visual analysis of more complex objects represented with two and more scalar fields describing various object properties. In this research, we consider a distance-based TF in Volume Rendering that high- 
lights features of interest mostly determined by the distance to reference structures (Tappenbeck et al., 2006). Those type of TFs takes advantage of Signed Signed Distance Field (SDF).

The Signed Distance Field (SDF) is a type of a scalar field, where the scalar value takes the value of a distance to the surface $d S$ of a solid object $S$ (Jones et al., 2006). The distance property of SDF allows for efficient ray-casting (Hart, 1996) and efficient computation of complex light-object interactions in optical model. Moreover, in this research, we will take advantage of the GPU implementation of the constructive approach for the entire dynamic volume scene modelling (Wang et al., 2011) based on SDF.

\subsection{Auditory Properties}

The auditory model often takes advantage of similarities in light and sound propagation (Takala and Hahn, 1992). The acoustic rendering equation (Siltanen et al., 2007) is a time-dependent version of the general rendering equation (Kajiya, 1986) that is a basis of the optical Volume Rendering models (Max and Chen, 2010). The sound propagation and auditory perception of this process to model auditory feedback (McArthur et al., 2017) are open research areas in augmented (Ribeiro et al., 2012) and virtual (Begault, 1994) reality. Auditory stimuli are wellknown tools in molecular structures interactive study (Liu and van der Heide, 2019) that is often considered as an alternative to haptic ones in the traditional area of their application. The reason is that it does not have to deal with haptic device limitations and allows for mapping and perception of a much wider range of properties (May et al., 2019).

The auditory study of volumetric structures as a part of data analysis is an open research area (Gionfrida and Roginska, 2017), (Malikova et al., 2019). The interpretation of sound parameters for the auditory analysis and control of the auditory representation of the phenomena can be not straightforward (Hermann, 2018). Thus, the research in the area mostly deals with the design of auditory mapping procedures for specific applications (Gionfrida and Roginska, 2017).

In this work, we take advantage of similarities in the light and sound propagation to introduce an auditory model based on sound wave generation as a result of impulse propagation through the object. Thus, we consider the ray-tracing technique for the simultaneous visual-auditory rendering of multi-scale phenomena.

We discuss auditory feedback as distance-based guidance or measurement procedure. For such pro- cedures design, we consider the physical process of sound propagation. Thus, the proposed auditory model allows natural perception and interpretation of the distance-dependent aspects of objects-sound wave interaction. An auditory guidance procedure can be used to inform of the spatial orientation and position while interacting with virtual objects. A measurement procedure allows tracking of small changes in features of dynamic phenomena. As the visual system can be fully engaged while performing a complex perception task, the additional source of information provided through other sensory stimuli is needed (Hou and Sourina, 2011). Thus, such auditory procedures are quite important.

The conceptual framework for the introduced procedures is based on sound propagation the digital waveguide approach to physically-based sound synthesis (Cook, 2002). We consider the use of a basic plucked string (Smith, 1992) model that allows auditory tracking of even the small changes in the distance of sound propagation with a pitch characteristic of sound.

\section{OUR APPROACH TO VISUAL-AUDITORY ANALYSIS OF MOLECULAR FIELDS}

Let us consider the case of the molecular structure that is a result of quantum simulation as the two main models are used for the phenomena analysis. The most common geometrical representation of molecular structures is the "Balls and Stick" model (Berg et al., 2002) (Fig. 2) (a). The model suggests that atoms are represented with spheres and atomic radii and distance between atoms approximate bonds between atoms and their types. Despite these simplifications, this model is good for measurements and monitoring of distance-related parameters.

Another model arises from quantum chemistry calculations and concentrates on the analysis of more complex abstraction for the representation of the molecular structure (Shusterman and Strauss, 2001) that usually suggests the necessity of simultaneous analysis of two scalar fields. The shape of the Electron density field is treated like molecule interaction boundary as it defines a probability of an electron occupying an infinitesimal element of space surrounding any given point. At the same time, its topological analyses provide a deeper understanding of bonding. The Electrostatic potential field represents a charge distribution, a property that allows predicting the behaviour of complex molecules. In the ideal case, the 


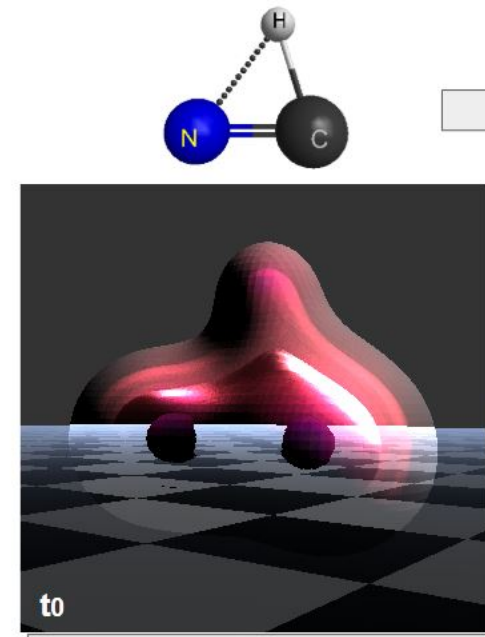

to

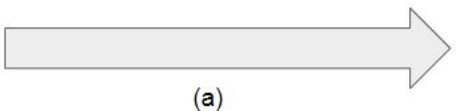

(a)

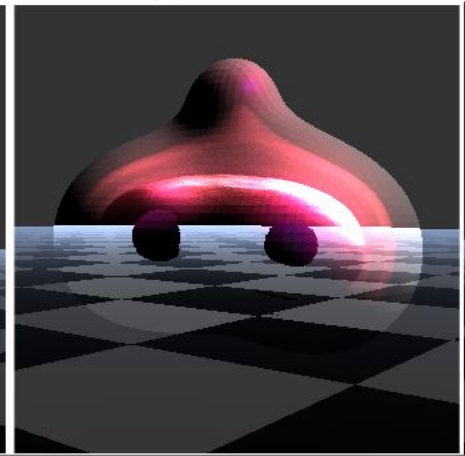

(b)

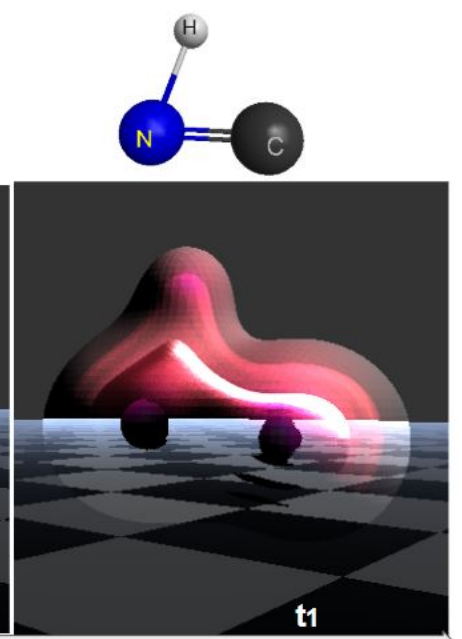

t1

Figure 2: Selected frames of dynamic Volume Rendering of the chemical transformation from HCN to the HNC isomer. Balls and Stick model (a) and quantum chemistry model (b).

user should be able to analyse both of those fields simultaneously (Shusterman and Strauss, 2001). Unlike balls and sticks, this model allows for more accurate analysis of the properties of a molecular phenomenon such as bonding, although its computation is expensive (see Fig. 2)(b)).

In our visual-auditory approach, we are employing both models in the analysis process keeping in mind the dynamic nature of the phenomena. As a case study, we analyse the dynamic reaction of the transformation of HCN to the HNC isomer (see Fig. 2). We employ dynamic Volume Rendering for simultaneous visual analysis of changes in both scalar fields of quantum chemistry model (see Fig. 2 (b)). We consider the auditory analysis as a well-proven tool for monitoring of small changes in dynamic characteristics and employ it for simultaneous measurements performed on the "Balls and Stick" model (see Fig. 2)(a). Below we will consider the HyperVolume model for the multi-scale representation of the phenomena and its further rendering to visual and auditory stimuli.

\subsection{HyperVolume Model}

We use a HyperVolume (HV) model to describe a studied phenomena through a tuple:

$m(t)=\left(G(t), A_{o}(t), A_{s}(t)\right):\left(F(X \mid t), S_{o}(X \mid t), S_{s}(X \mid t)\right)$

where $G(t)$ is dynamic geometry described with function $F(X \mid t) ; S_{o}(X \mid t)$ and $S_{S}(X \mid t)$ are point attribute functions $S$ defining optical $A_{o}$ and auditory $A_{s}$ properties of heterogeneous object.
Thus, we define a vector-valued function. The first component $F$ is responsible for the object geometry $G$. In our approach $F$ is an SDF, which is obtained from the input scalar field by using signed distance transform (Jones et al., 2006). The auditory properties in the form of the generated sound wave are defined with $S_{s}(X)$. The attribute $S_{o}(X)$ defines the results of mapping to the optical properties such as colour and opacity. Note that in the general case attributes $S$ are not scalars but vectors. For example, the colour and transparency information in $S_{o}$ is normally represented as a four-component RGBA value.

In the case of the molecular structure, the dynamic geometry $G(t)$ is represented with electron density field isosurface as it is interpreted as a molecule interaction boundary. The optical model $S_{o}(X)$ is used for visual analysis of the quantum chemistry phenomena representation. The auditory model $S_{S}(X)$ is used to judge about the dynamic change of measured characteristics performed on the basic "Ball and Sticks" model. In this case study, we consider its use for bond length measurement.

The SDF-based ray-tracing is used for dynamic visual-auditory rendering of multi-scale objects. We use the constructive approach for modelling $S_{o}(X \mid t)$ and $S_{S}(X \mid t)$ attributes of dynamic object $m(t)$ (Pasko et al., 2001) with SDF based geometry representation for $F(X \mid t)$ and implement most of visual-auditory analysis pipeline on GPU (see section 4). Below we consider optical and auditory models in more detail. 


\subsection{Optical Model for Ray-casting}

The main target of the optical model $S_{o}(X)$ in equation 3 is to allow the user getting insight into the molecular phenomena through colour and opacity. To address the problem of complex phenomena represented with two scalar fields (Electron density and Electrostatic potential) visualisation, we propose a custom distance-based TF, that operates those two fields simultaneously. We consider optical models described with equations 1 and 2 for visual analysis quality enhancement.

The emission-absorption 1 based $S_{o}(x)$ was not particularly efficient. We had to adjust the transparency value to handle dark regions that made the model look like a set of coloured isosurfaces (see Fig.3(a)). The single scattering with a colour blending allowed assessing both changes of the Electron Density isosurface shape and the Electrostatic Potential distribution (see Fig.3(b)).

The results of conventional Volume Rendering for various models $S_{o}(x)$, we have suggested for $\mathrm{HCN}$ molecule case study, are presented in Fig. 3. Below we will describe the proposed distance-based TF. The use of SDF allows for optical model accelerated computation of lightning and scattering.

\subsubsection{Transfer Function}

To define a distance-based TF, we consider the BlinnPhong model and use the SDF object geometry definition. The proposed TF highlights the shape of Electron Density Field and to allows judging on the value of the Electrostatic Potential Field. We employ the specular and diffuse shading to provide highlights of both scalar fields and especially closer to the "atom" regions.

As described in (Levoy, 1988), a numerical optical model for colour at i-th voxel $C_{i}=C\left(x_{i}\right)$ for a parallel light source is:

$$
\begin{aligned}
C_{i}=c_{p} \cdot k_{a}+ & \frac{c_{p}}{k_{1}+k_{2} \cdot d\left(x_{i}\right)} . \\
& {\left[k_{d}\left(N \overrightarrow{\left(x_{i}\right)} \cdot \vec{L}\right)+k_{s}\left(N \overrightarrow{\left(x_{i}\right)} \cdot \vec{H}\right)^{n}\right] }
\end{aligned}
$$

where $N \overrightarrow{\left(x_{i}\right)}$ is a surface normal at voxel $x_{i}$ location, $\vec{L}$ is a normalised vector in the direction of the light source, $\vec{H}$ is a normalised vector in the direction of maximum highlight; $c_{p}$ is a component of colour parallel to the light source; $k_{1}, k_{2}$ are constants used in a linear approximation of the depth-queuing; $d\left(x_{i}\right)$ is perpendicular distance from the picture plane to the voxel location $x_{i}$.

The ambient, diffuse, specular coefficients $k_{a}, k_{d}$, $k_{s}$ are vary at different parts of the volume and are

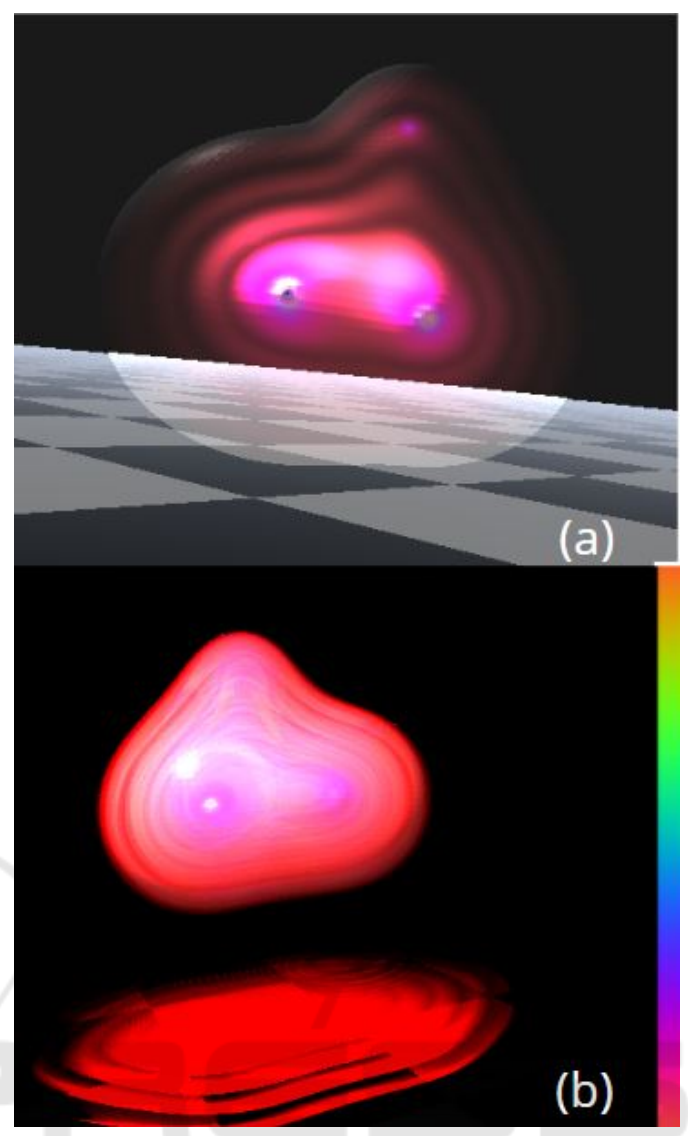

Figure 3: Volume Rendering of the HCN molecule. Rendering with introduced TF and the following optical models: a) without scattering; b) with basic single scattering.

controlled by the SDF value of the Electron Density Field in order to highlight how colour is distributed on selected isosurfaces. We multiply the opacity value of the TF by the coefficient controlling the isosurface highlight $o_{h}: o_{h}=o_{0}^{\frac{d\left(x_{i}\right)}{d_{\max }}} \cdot\left|\frac{d\left(x_{i}\right)}{d_{\max }} \cdot \pi \cdot f\right|$, where $f$ is a number of isosurfaces; $d_{\max }$ is a maximum value of the distance field; $o_{0}$ is a basic coefficient that is the maximum $o_{h}$ value can take. If $o_{h}$ is greater than a certain threshold value we apply surface shading with $k_{s}$ and $k_{d}$ values equal to $o_{h}$.

\subsection{Auditory Model for Ray-casting}

The proposed approach allows the visual-auditory analysis of both quantum chemistry and "Balls and Sticks" representations. The optical property $S_{o}(X)$ allows judging on phenomena through colour and opacity. The sound perception has the potential to provide a wider range of auditory entities for interpretation such as pitch, volume, duration, rhythm, etc. We employ the physical process of sound propaga- 
tion to directly construct a sound wave representation. We consider the task of auditory analysis through the pitch of the dynamic length changes in $\mathrm{NC}$ bond for the dynamic chemical reaction of transformation from the $\mathrm{HCN}$ to the $\mathrm{HNC}$ isomer.

The atoms of "Balls and Sticks" model are represented as SDF spheres $S p_{h}, S p_{c}, S p_{n}$ with sufficiently small radii. We take advantage of an ideal string model to render distance property of $\mathrm{NC}$ bond to sound wave and judge about the bond length through the pitch. The process is schematically presented in Fig. 4 and is described as follows.

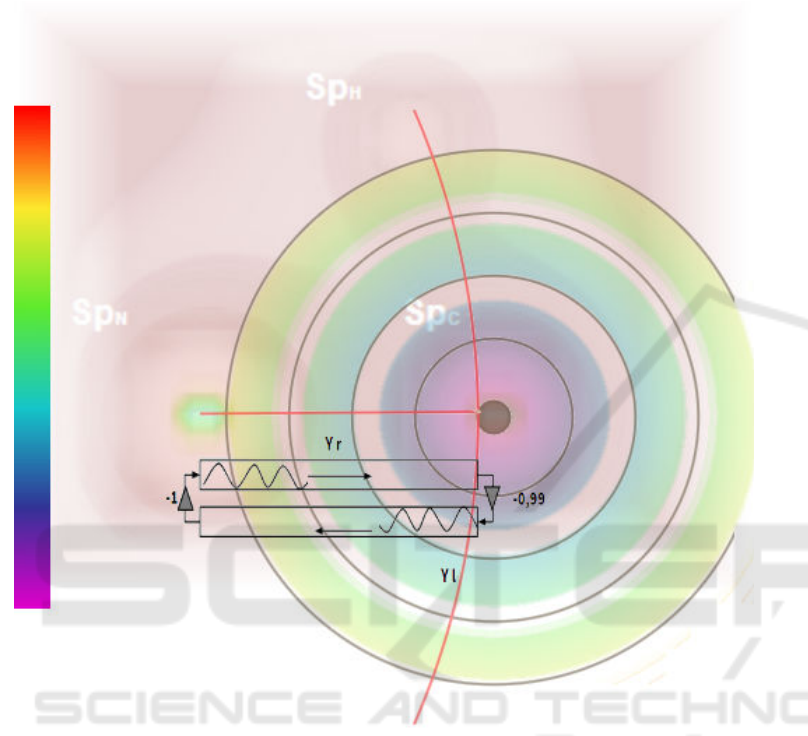

Figure 4: Rendering to auditory stimuli. Schematic representation of ray-casting the length of NC bond and mapping it into the sound with the ideal string model.

We adapt the travelling wave solution for an ideal plucked string (Smith, 1992) for introduced auditory model. The result of an initial impulse of the plucked string travelling wave $y(x, t)$ can be represented as a sum of the right going $y_{r}(x-c t)$ and left going $y_{l}(x+$ $c t$ ) waves (see Fig. 4 (b)).

The travelling waves are simulated through the delay lines. Once the initial waves are generated as a result of a string picking, they travel along the string. Thus, the time impulse needs to pass the distance till it is reflected. The propagation process is aurally perceived through the pitch: $l=2 * R / p$, where $R$ is a sampling rate, string length $l$ and $p$ is a perceived pitch. We perform auditory analysis to judge on small changes and we use the ray-casting procedure to compute the sound wave.

\section{IMPLEMENTATION}

The HV model is described through a construction tree. The leaves are initial scalar fields data loaded to GPU memory as 3D textures, and the nodes are operations similar to the initial idea presented in (Pasko et al., 2001). The conventional TF is considered as the "node of operation" within the construction tree of the HV model.

We take advantage of the modern CUDA graph API (NVIDIA, 2019) to describe such construction trees and render them to textures on-demand with the updated parameters. The final 3D textures represent the HV model $m(t)$ and the lighting conditions. Those textures are sampled during the conventional Volume Rendering and auditory feedback computation (see the example in Fig. 5). The GPU data flow model allows integration of image processing techniques that can be implemented as graph nodes applied to texture volumes with CUDA graph API. Currently, we have considered a basic case of "host" implementation with Image Processing Toolkit (ITK) (Johnson et al., 2015), to extract the centres of SDF spheres $S p_{h}, S p_{c}, S p_{n}$ that represent the atoms of the molecule from scalar fields.

The final visual-auditory scene is presented as a set of objects $S=\left(H V, S D F_{o}, L\right)$, where $\mathrm{HV}$ are HyperVolume representations in a form of a tuple described above; $S D F_{o}$ is a set of the SDF geometry in the scene; $L$ are the precomputed lighting conditions for the scene. This representation naively supports further extension with interactive procedures that provide auditory feedback. The further extension can be introduced on the same basis of SDF geometry modelling and ray tracing.

The different parts of the scene in Fig. 5 representation are evaluated in advance, stored as textures and updated on-demand (see section 2.1 for details). The proposed approach handles the rendering of both surfaces and volume objects in a similar way. Thus, the entire scene is procedurally modelled, stored and rendered on GPU. Below we describe the obtained results for dynamic visual-auditory molecular phenomena study.

\section{RESULTS}

We analyse the dynamic reaction of the transformation of $\mathrm{HCN}$ to the HNC isomer (see Fig. 2) through the obtained visual-auditory image of the phenomena. The initial data is a sample data from the GAMESS software package (M.W.Schmidt et al., 1993) that describes an HCN initial saddle point state before the 



$m(t)=(G(t), S o(t), S s(t))$
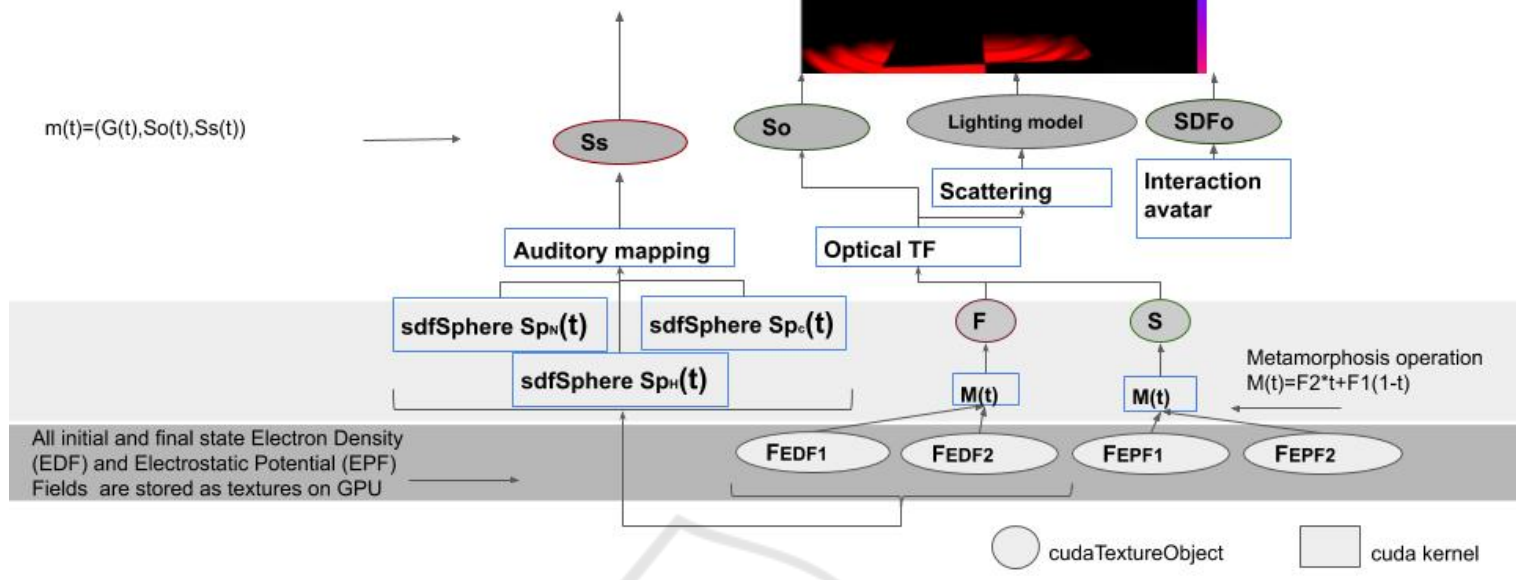

Figure 5: The CUDA implementation scheme of the HyperVolume model construction tree for the the process of $\mathrm{HCN}$ to the $\mathrm{HNC}$ isomer reaction.

HNC isomer reaction and the final state - HNC isomer (see Fig.2(a,b)). The results of visual-auditory rendering are presented in the accompanying video. We use the auditory feedback for measurements performed on the molecular structure.

As an example, we use it to highlight a process of small changes of $\mathrm{CN}$ bond length in $\mathrm{HNC}$ isomer reaction dynamic process (Fig. 2(a)) and judge about the dynamic change of distance through the pitch. The process is schematically presented in Fig. 4 (b). The pitch changes from lower to higher values as the distance becomes smaller. At the same time, we judge visually on dynamic change within quantum chemistry representation of the molecular phenomena through the dynamic Volume Rendering.

\section{CONCLUSIONS AND DISCUSSION}

This paper presents a novel approach to the scientific visualisation of complex physical phenomena such as dynamic molecular fields based on the visual-auditory Volume Rendering. The proposed solution is based on the techniques used for multi-scale, heterogeneous objects modelling and rendering. The main features of the approach are HyperVolume model for visualauditory scene representation and ray-casting to visual and auditory stimuli.
We have addressed a particular problem of analysis of molecular fields that are results of quantum chemistry simulation. Taking this case study as an example, we have demonstrated how the visual-auditory analysis based on the presented approach can be used to get insight into dynamic multi-scale phenomena as solely visual analysis can be challenging. We have implemented our approach on GPU, demonstrated its flexibility and considered the representative case studies. The approach allows GPU data flow for visualauditory Volume Rendering.

The SDF technique has applications in image processing tasks and is an essential part of computationally expensive interactive procedures, like collision detection. The proposed approach has the potential to combine the heterogeneous objects modelling procedures and the conventional image processing on GPU. The proposed approach further extension will also consider the interactive visual-auditory rendering tasks that target the enhancement of molecule editing and other interactive exploration procedures.

So far we have considered the basic optical model and have taken into account only single scattering. Rendering of more complex auditory and optical models is the area of future research. Exploring multiscale object shapes and internal properties through the perception of the spatial 3D sound is on our agenda. 


\section{REFERENCES}

Begault, D. R. (1994). 3D Sound for Virtual Reality and Multimedia. Academic Press Professional, Inc., San Diego, CA, USA.

Berg, J. M., Tymoczko, J. L., and Stryer, L. (2002). Appendix: Depicting Molecular Structures. New York: W. H. Freeman.

Cook, P. R. (2002). Real Sound Synthesis for Interactive Applications. A. K. Peters, Ltd., Natick, MA, USA.

Gionfrida, L. and Roginska, A. (2017). A novel sonification approach to support the diagnosis of alzheimer's dementia. Frontiers in Neurology, 8:647.

Hart, J. C. (1996). Sphere tracing: a geometric method for the antialiased ray tracing of implicit surfaces. The Visual Computer, 12(10):527-545.

Hermann, T. (2018). Wave space sonification. In ICAD 2018, pages 49-56.

Hermann, T., Hunt, A., and Neuhoff, J. G., editors (2011). The Sonification Handbook. Logos Publishing House, Berlin, Germany.

Hou, X. and Sourina, O. (2011). Six degree-of-freedom haptic rendering for biomolecular docking. Transactions on Computational Science, 12:98-117.

Jönsson, D., Sundén, E., Ynnerman, A., and Ropinski, T. (2014). A survey of volumetric illumination techniques for interactive volume rendering. Computer Graphics Forum, 33(1):27-51.

Johnson, H. J., McCormick, M. M., and Ibanez, L. (2015).

The ITK Software Guide Book 1: Introduction and Development Guidelines - Volume 1. Kitware, Inc., USA.

Jones, M. W., Bærentzen, A., and Šrámek, M. (2006). 3d distance fields: A survey of techniques and applications. IEEE Transactions on Visualization and Computer Graphics, 12(4):581-599.

Kajiya, J. T. (1986). The rendering equation. SIGGRAPH Comput. Graph., 20(4):143-150.

Kniss, J., Premoze, S., Hansen, C., Shirley, P., and McPherson, A. (2003). A model for volume lighting and modeling. IEEE Transactions on Visualization and Computer Graphics, 9(2):150-162.

Levoy, M. (1988). Display of surfaces from volume data. IEEE Comput. Graph. Appl., 8(3):29-37.

Liu, D. and van der Heide, E. (2019). Interactive auditory navigation in molecular structures of amino acids: A case study using multiple concurrent sound sources representing nearby atoms. In ICAD 2019, Newcastle upon Tyne, UK., pages 140-146. Georgia Institute of Technology.

Lodha, S. K., Beahan, J., Heppe, T., Joseph, A. J., and ZaneUlman, B. (1997). Muse: A musical data sonification toolkit. In ICAD1997, Palo Alto, California, page 36-40. Georgia Institute of Technology.

Malikova, E., Adzhiev, V., Fryazinov, O., and Pasko, A. (2019). Visual-auditory volume rendering of scalar fields. In ICAD 2019, Northumbria University, Newcastle upon Tyne, UK. Georgia Institute of Technology.
Max, N. and Chen, M. (2010). Local and global illumination in the volume rendering integral. In Scientific Visualization: Advanced Concepts, pages 259-274.

May, K. R., Sobel, B., Wilson, J., and Walker, B. N. (2019). Auditory displays to facilitate object targeting in $3 \mathrm{~d}$ space. In ICAD 2019, Newcastle upon Tyne, UK. Georgia Institute of Technology.

McArthur, A., Sandler, M., and Stewart, R. (2017). Distance in audio for vr: Constraints and opportunities. In Proceedings of the 12th International Audio Mostly Conference on Augmented and Participatory Sound and Music Experiences, AM '17, pages 28:1-28:7, New York, NY, USA. ACM.

M.W.Schmidt, K.K.Baldridge, J.A.Boatz, S.T.Elbert, M.S.Gordon, J.H.Jensen, S.Koseki, N.Matsunaga, K.A.Nguyen, S.Su, T.L.Windus, M.Dupuis, and J., J. (1993). General atomic and molecular electronic structure system. Comput. Chem., 14:1347-1363.

NVIDIA (2019). CUDA C++ Programming Guide.

Pasko, A., Adzhiev, V., Schmitt, B., and Schlick, C. (2001). Constructive hypervolume modeling. Graphical Models, 63(6):413-442.

Ribeiro, F., Florêncio, D., Chou, P. A., and Zhang, Z (2012). Auditory augmented reality: Object sonification for the visually impaired. In 2012 IEEE 14th International Workshop on Multimedia Signal Processing (MMSP), pages 319-324.

Schlegel, P., Makhinya, M., and Pajarola, R. (2011). Extinction-based shading and illumination in gpu volume ray-casting. IEEE Transactions on Visualization and Computer Graphics, 17(12):1795-1802.

Shusterman, A. and Strauss, L. (2001). Teaching chemistry with electron density models. 2. can atomic charges adequately explain electrostatic potential maps? The Chemical Educator, 6:36-40.

Siltanen, S., Lokki, T., Kiminki, S., and Savioja, L. (2007). The room acoustic rendering equation. 122:1624.

Smith, J. O. (1992). Physical modeling using digital waveguides. Computer Music Journal, 16(74):74-91.

Takala, T. and Hahn, J. (1992). Sound rendering. In Proceedings of the 19th Annual Conference on Computer Graphics and Interactive Techniques, SIGGRAPH '92, pages 211-220, New York, NY, USA. ACM.

Tappenbeck, A., Preim, B., and Dicken, V. (2006). Distance-based transfer function design: Specification methods and applications. In SimVis2006, pages 259274

Wahle, M. and Wriggers, W. (2015). Multi-scale visualization of molecular architecture using real-time ambient occlusion in sculptor. In PLoS Computational Biology, volume 11.

Wang, L., Yu, Y., Zhou, K., and Guo, B. (2011). Multiscale vector volumes. ACM Trans. Graph., 30(6):167:1167:8. 